\title{
ENSAIO CLÍNICO COM ETOFAMIDA * NO TRATAMENTO DA AMEBIASE INTESTINAL CRÔNICA EM CRIANÇAS **
}

\author{
Donald Huggins *** e Marluce F. Maciel ****
}

\begin{abstract}
Os AA. trataram 40 crianças portadoras de amebiase intestinal crônica, cujas idades variaram entre dois (três casos) a seis anos (cinco doentes), com Etofamida na apresentação de suspensão (cada $5 \mathrm{ml}$ contém $100 \mathrm{mg}$ da substância ativa), na dose de $100 \mathrm{mg}$ cinco vezes ao dia e durante três dias consecutivos (dose total de 1,5 g). O controle de cura parasitológica foi realizado pelas técnicas de Faust e cols. e de Hoffman, Pons e Janer no 10., $15 .^{\circ}$ e 25. dias após o tratamento, sendo também usada a hematoxilina férrica neste último controle. Obtiveram os AA. 90\% de cura parasitológica (36 enfermos) $e$ excelente tolerância ao medicamento.
\end{abstract}

\section{INTRODUÇÃO}

A amebíase intestinal em sua forma crônica ocupa um papel fundamental no desencadeamento de diarréias em crianças. Sua prevalência é bastante elevada em nosso meio, sobretudo em individuos de baixa condição sócio-econômica, especialmente os enfermos que procuram tratamento ambulatorial no Hospital das Clínicas da F.M.U.F., PE.

Em se tratando de uma parasitose intestinal de considerável importância, não somente pela sua alta prevalência, como também pelas complicações que poderá determinar: enterorragias, necrose hepática (abcesso amebiano), etc., têm suscitado dos pesquisadores da Indústria Farmacêutica a procura de novas substâncias para sua erradicação, sem trazer manifestações colaterais indesejáveis durante sua administração.

Continuancio na sua linha de pesquisa, em busca de drogas antiparasitárias, de eficácia reconhecida, o Laboratório Carlo
Erba do Brasil S/A sintetizou através da clorfenoxamida (Clefamida - Mebinol) um potente amebicida - Etofamida, comprovado experimentalmente por De Carneri e cols. (5 e 6) e, posteriormente, no homem por diversos investigadores $(1,2,3$, $4,7,8,9,10,11,12$ e 13). Em trabalhos anteriores estudamos a droga em adultos (7 e 8) e atualmente empreendemos nova pesquisa, agora em crianças, com o intuito de aprofundarmos nossos conhecimentos com relação à referida substância.

\section{MATERIAL E MÉTODOS}

Medicamos 40 crianças com a forma crônica da amebiase intestinal no Ambulatório das Disciplinas de Puericultura e Doenças Infectuosas e Parasitárias da F.M.U.F., PE, de ambos os sexos, com idades variáveis entre dois (três enfermos) a seis anos (cinco doentes) e peso compreendido entre $10 \mathrm{~kg}$ (dois casos) a $25 \mathrm{~kg}$ (três pacientes). A substância administrada (Etofamida) é quimicamente a $\mathrm{N}$-beta-

* KITNOS: Carlo Erba do Brasil S/A, São Paulo.

* Disciplinas de Doencas Infectuosas e Parasitá rias e de Puericultura da F.M.U.F. PE.

*** Pros. Adjunto.

**** Auxiliar de Ensino.

Recebido para publicaçã,o em 13-1-75. 
-etoxi-etil-N-para-fenoxi-4-nitro-benzil-dicloroacetamida e apresentada na forma de suspensão, contendo, em cada $5 \mathrm{ml}, 100 \mathrm{mg}$ da substância ativa e a dose empregada foi de $500 \mathrm{mg}$ ao dia (fracionada em duas tomadas após o desjejum e almoço e uma medida depois do jantar) durante três dias consecutivos (dose total: $1,5 \mathrm{~g}$ ).

o diagnóstico da parasitose intestinal foi realizado pelas técnicas de Faust e cols. e Hoffman, Pons e Janer com sedimentação espontânea em água durante 24 horas. Após o tratamento, utilizamos como critério de cura parasitológica a negativação dos exames parasitológicos das fezes em três ocasiōes (controles) sucessivas, efetuadas no $10 .^{\circ}, 15 .^{\circ}$ e $25 .^{\circ}$ dias, usando-se os mesmos métodos já citados e também o da Hematoxilina férrica apenas no último controle. Antes e após a terapêutica, realizamos paralelamente os seguintes exames laboratoriais: hemograma, glicemia, creatinina, uréia, bilirrubinas, transaminases e sumário de urina.

\section{RESULTADOS}

Ao final da investigação conseguimos obter cura parasitológica definitiva em 36 pacientes $-90 \%$ dos casos medicados, enquanto os restantes doentes (quatro) continuaram eliminando cistos de Entamoeba histolytica (ver Quadro). A tolerância ao medicamento foi considerada excelente, tanto do ponto de vista clínico, como bioquímico, em virtude de não se constatar nenhuma manifestação colateral ou desvio do padrão normal das provas laboratoriais empregadas.

\section{COMENTÁRIOS}

As pesquisas levadas a cabo em individuos adultos, portadores de amebiase in- testinal crônica, demonstraram que a nóvel substância amebicida apresentava intensa atividade contra o protozoário. Assim,

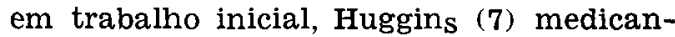
do 22 pacientes (21 adultos e uma criança), obteve $95,4 \%$ de cura parasitológica e ótima tolerância.

Salles, Viana e Costa (11) trataram 82 adultos e 85 crianças com Etofamida, em dois esquemas terapêuticos: no primeiro, empregaram a dose de $600 \mathrm{mg}$ por dia, durante cinco cias para os adultos e $20 \mathrm{ml}$ a.o dia durante cinco dias (xarope a $2 \%$ ), obtendo $82,5 \%$ e $77,8 \%$ de cura parasitológica, respectivamente para adultos e crianças. No segundo esquema, usaram $900 \mathrm{mg}$ por dia durante cinco dias para adultos e $30 \mathrm{ml}$ por dia também por cinco dias para as crianças, conseguindo $100 \%$ de cura parasitológica apra os adultos e $88,2 \%$ para as crianças. A tolerância ao medicamento foi satisfatória, apenas relatando náuseas e meteorismo em poucos casos.

Aboim, Iório e Ramos (1) trataram 50 enfermos acultos, conseguindo cura parasitológica global em torno de $94 \%$.

Vasconcellos (13), administrando a 20 doentes portadores de amebíase intestinal (14 casos crônicos e seis agudos), a substância em tela, conseguiu em uma primeira série de tratamento $80 \%$ de cura parasitológica e $95 \%$ com uma segunda série cie tratamento. Efeitos secundários foram observadcs em apenas três pacientes (náuseas em dois e cefaléia em um).

Salle $_{S}$ e Leitão (12) medicaram dois grupos de pacientes constituídos cada por 46 enfermos, com a dose de 450 e $600 \mathrm{mg}$ por dia cuurante cinco dias, respectivamente para o primeiro e segundo grupo, obtiveram $86,9 \%$ e $93,4 \%$ de cura parasitológica. Salientaram que as reações secundárias referidas durante o período de tratamento foram de pouco significado clínico.

Resultados obtidos com Etofamida (suspensão) no tratamento da amebiase intestinal crônica em crianças

\begin{tabular}{c|c|c|c}
\hline $\begin{array}{c}\text { N. CASOS } \\
\text { TRATADOS }\end{array}$ & DOSE & $\begin{array}{c}\text { PACIENTES } \\
\text { CURADOS } \\
\text { N. }\end{array}$ & $\begin{array}{c}\text { EFFITOS } \\
\text { COLATERAIS }\end{array}$ \\
\hline 40 & $\begin{array}{c}500 \mathrm{mg} \mathrm{x} \text { dia } \mathrm{x} \\
\text { três dias }\end{array}$ & $36-90 \%$ & NENHUM \\
\hline
\end{tabular}


Moura (9), estudando 18 crianças, nove medicadas com Etofamida e nove com placebo, verificou 24 horas após o tratamento que $88 \%$ dos pacientes que receberam a substância em tela, negativaram os exames parasitológicos das fezes, enquanto $64 \%$ dos que receberam o placebo apresentaram exames parasitológicos das fezes negativos. Cerca de 10 dias após a terapêutica, dos oito casos negativos, apenas cinco compareceram para novo controle laboratorial, sendo negativos em todos os exames parasitológicos. Ao fim de 30 dias, apenas uma criança retornou para novo controle de cura, continuando ainda o exame negativo.

Botero e Cols. (3) utilizaram a droga como medida profilática em uma comunidade na qual a prevalência inicial da amebíase era de aproximadamente $29 \%$. Em um grupo de 100 indivíduos submetidos anteriormente ao tratamento curativo e posteriormente ao profilático, com uma dose de $500 \mathrm{mg}$ semanais, durante um ano, verificaram a negativação em $100 \%$ dos casos, enquanto o grupo medicado com placebo, a prevalência inicial elevou-se para $40 \%$ ao fim cie um ano.

Daher (4) tratou 92 pacientes, os quais foram divididos em dois grupos:

Grupo 1 - Composto de 40 crianças, as quais receberam Etofamida na forma de suspensão a $2 \%$ na seguinte posologia:

a) cinco colheres medidas ao dia por três dias, em 20 crianças;

b) três colheres medidas ao dia, durante cinco dias, também em 20 crianças.

Grupo 2 - Constituído por 52 enfermos (40 adultos e 12 crianças), que tomaram as seguintes doses: a) em 11 doentes administrou 1.000 $\mathrm{mg}$ ao ciia por três dias;

b) em quatro crianças empregou $a$ dose de $800 \mathrm{mg}$ por dia, durante dois dias;

c) em 37 pacientes usou doses mais elevadas: $2.000 \mathrm{mg}$ ao dia, durante dois dias, em seis doentes; $1.600 \mathrm{mg}$ ao dia, durante dois dias, em 31 enfermos.

Verificou nos pacientes medicados e independentemente da dose empregada, cura parasitológica em todos os casos, ou seja, $100 \%$.

Baranski (2) tratou 20 pacientes adultos portadores de amebíase intestinal crônica, com Etofamida na dose de $1,0 \mathrm{~g}$ por dia (cinco comprimidos de $200 \mathrm{mg}$ ) durante três dias. Obteve cura parasitológica de $90 \%$ com a primeira série de tratamento e $100 \% \mathrm{ccm}$ um segundo curso do medicamento. Reações colaterais foram verificadas em raros casos, tais como cólicas abdominais, diarréia, cefaléia e insônia, com regressão espontânea a curto prazo.

Huggins (8), em trabalho anterior, medicou 51 enfermos portadores de amebíase intestinal crônica com Etofamida na dose diária de $1,0 \mathrm{~g}$ (cinco comprimidos de 200 $\mathrm{mg}$ ) durante três dias consecutivos, obtencio $92,16 \%$ de cura parasitológica e ausêrncia de efeitos colateriais.

Recentemente, Otero e Cols. (10) trataram 50 doentes com amebíase intestinal crônica com Etofamida, na dose de $1,0 \mathrm{~g}$ por dia, durante três dias consecutivos e paraleiamente pesquisaram a função hepática durante a terapêutica. Obtiveram $90 \%$ cie cura parasitológica e salientaram a inocuidade do produto sobre o hepatocito.

\section{$S U M M A R Y$}

A new antiamebic agent, Ethophamide, was tried in the treatment of 40 children suffering chronic intestinal amebiasis, of both sexes, and aged from two (three patient) to six years old (five patients).

The drug was given in oral suspension containing $2 \%$ of Ethophamide, and the dosage used was $500 \mathrm{mg}$ daily for three days. Laboratorial controls were made before the treatment and repeated 10, 15, and 25 days after its completion, and consisting of Faust \& cols., Hoffman, Pons and Janer mehods, and iron hematoxylin (on the 25th day).

After the treatment 36 patients (or 90\%) were cured, and the tolerance to medication was considered very good. In the light of the excellent results obtained, the Authors concludes that Ethophamide could be the drug of choice for the treatment of amebiasis. 


\section{REFERÊNCIAS BIBLIOGRÁFICAS}

1. ABOIM, E., IÓRIO, A. \& RAMOS J.L.S. - Do tratamento da amebíase intestinal. Eticlordifene. Hospital, 76: 205-225, 1969.

2. BARANSKI, M.C. - Tratamento da amebíase intestinal crônica pela Etofamida. Tribuna Médica, 14: 35, 1971.

3. BOTERO, D., ROJAS, W., HEYES, D. \& SANCHEZ, M.A. - Estudio epidemiologico, terapeutico y quimioprofilatico de amebiasis intestinal en el Municipio de Apartado (Colômbia). Antioquia Médica, 21: 217-228, 1971.

4. DAHER, H.R. - A etofamida no tratamento da amebiase intestinal. Tribuna Médica, 14: 3\$-40, 1971.

5. DE CARNERI, I. \& ALII - Un nuevo antiamébico: El Kitnos, Eticlordifene. Giorn. Mal. Infett. Parassit., 21: 281-283, 1969.

6. DE CARNERI, I. \& ALII - Ethychlordiphene: A new antiamebic agent. Proc. 6th International Congress of Chemotherapy, Tokyo (Kyoto), Japan, August 10th to 15th, 1969.

7. HUGGINS, D. - Tratamento da amebiase intestinal crônica com Eticlordifene. Hospital, 75: 2.011-2.019, 1969.
8. HUGGINS, D. - Ensaio clínico com Etofamida no tratamento da amebiase intestinal crônica. (Estudo com novo esquema terapêutico). Tribuna Médica, 14: 42-50, 1971.

9. MOURA, $\mathrm{H}$. -Relatório fornecido ao Laboratório Carlo Erba do Brasil, S/A, 1968.

10. OTERO, N.B. ET ALII - Ação da Etofamida na amebíase intestinal crônica e sua inocuidade sobre o hepatocito. Revta. Bras. Clin. Terap., 2: 581-586, 1973.

11. SALLES, J.M., VIANA, M.T.M. \& COSTA, C.A. - Avaliação da atividade do Eticlordifene na amebiase intestinal. Hospital, 75: 2.189-2.194, 1969 .

12. SALLES, J.M. \& LEITÃO, E.G. Tratamento da amebíase intestinal com Eticlordifene. Hospital, 77: 2.073$-2.080,1970$.

13. VASCONCELLOS, D. - Experiência com novo amebicida Eticlordifene. Hospital, 76: 333-1.335, 1969. 